\title{
Geomagnetic activity recurrences for predicting the amplitude and shape of solar cycle n. 25
}

\author{
Piero Diego* (1) and Monica Laurenza (1) \\ INAF-Istituto di Astrofisica e Planetologia Spaziali, Via del Fosso del Cavaliere, 100, 00133 Roma, Italy
}

Received 26 February 2021 / Accepted 7 September 2021

\begin{abstract}
Predicting solar activity is one of the most challenging topics among the various Space Weather and Space Climate issues. In the last decades, the constant enhancement of Space Climate data improved the comprehension of the related physical phenomena and the statistical bases for prediction algorithms. For this purpose, we used geomagnetic indices to provide a powerful algorithm (see Diego et al. [2010. J Geophys Res 115: A06103]) for the solar activity prediction, based on evaluating the recurrence rate in the geomagnetic activity. This paper aims to present the validation of our algorithm over solar cycle n. 24, for which a successful prediction was made, and upgrade it to forecast the shape and time as well as the amplitude of the upcoming cycle n. 25. Contrary to the consensus, we predict it to be quite high, with a maximum sunspot number of $205 \pm 29$, which should be reached in the first half of 2023 . This prediction is consistent with the scenario in which the long-term Gleissberg cycle has reached its minimum in cycle n. 24 , and the rising phase is beginning.
\end{abstract}

Keywords: space weather / Sun / solar-terrestrial relations / geomagnetic activity

\section{Introduction}

The solar cycle (SC), or the Schwabe cycle, is a periodic variation of the activity level on the Sun over a period of about 11 years. Analyses of historical data on sunspots and related solar activity parameters have revealed a wealth of information on the solar cycle, although accurate advanced prediction of the cycle features, such as the shape and amplitude, is challenging. Nevertheless, the solar cycle prediction is important to test our knowledge of the solar dynamo and gauge the potential impact of space weather in the upcoming cycle, as the solar cycle can give a rough idea of the frequency of space weather storms.

A variety of methods has been developed to predict the solar cycle amplitude (see Pesnell, 2008; Petrovay, 2020), mainly based on the underlying physics of sunspot production, such as solar surface flux transport and/or solar dynamo processes (e.g., Charbonneau, 2010; Cameron et al., 2017; Bhowmik \& Nandy, 2018; Upton \& Hathaway, 2018), or precursor quantities of solar activity or magnetism (e.g., Schatten, 2005; Svalgaard et al., 2005; Pesnell \& Schatten, 2018). For instance, the polar magnetic field precursor method, as first suggested by Schatten et al. (1978), relies on the correlation between the amplitude of a sunspot maximum with a measure of the amplitude of the magnetic field near the Sun's poles at the preceding cycle minimum (e.g., Schatten, 2005; Svalgaard et al., 2005).

*Corresponding author: piero.diego@inaf.it
One class of precursors that have proven to be effective in predicting the solar cycle uses geomagnetic activity indices during the preceding cycle or near the time of minimum as an indicator of the amplitude for the next cycle. Ohl (1966) found that the minimum level of geomagnetic activity seen in the $a a$ index near the time of sunspot cycle minimum was a good predictor for the amplitude of the next cycle. Further modifications (Feynman, 1982; Hathaway \& Wilson, 2006) pointed out that the component of geomagnetic activity due to recurrent high-speed solar wind streams (HSSs) is a very good indicator for the amplitude of the following sunspot cycle. In addition, Diego et al. (2010), studying the persistence of the HSSs recurrence through their effects on geomagnetic activity (see also Sargent, 1985; Levitin et al., 1995), found a significant correlation between the average levels of the recurrence index during the descending phase of each solar cycle with the sunspot area (SA) integrated over the subsequent cycle, thus providing a new method for cycle strength prediction. In more detail, Diego et al. (2010) performed several attempts to identify the most suitable index and provided information on long-lasting recurrent HSSs, related to long-lived corotating regions on the Sun by improving Levitin's method. The most important advance of the suggested index relies on its variation for increasing number of Bartels rotation, as described in the following section. Multiple forecasts were made for solar cycle 24 (SC 24) with little consensus. A summary and analysis of more than 100 predictions of the amplitude of the past SC 24 were presented by 
Pesnell (2016), ranging from zero to unprecedented levels of solar activity (see also Pesnell, 2008, 2018). SC 24 turned out to be a below-average amplitude, peaking at an annual sunspot number (SN) of about 113. The polar field precursor method and the Diego et al. (2010) one proved to be particularly accurate for SC 24, although the latter provided a prediction for the sunspot areas instead of sunspot number. Generally, precursor methods provided better predictions for SC 24 with respect to dynamo-based ones. Strong criticism of this kind of prediction was provided by Tobias et al. (2006) and Bushby \& Tobias (2007), concluding that the solar dynamo is deterministically chaotic and thus inherently unpredictable. On the other hand, the problem with precursor methods is the limited advanced prediction, which makes them more useful for Space Weather than Space Climate.

As far as the new cycle 25 is concerned, the solar cycle 25 prediction panel has reached a consensus that SC 25 will be similar in size to SC 24, the maximum occurring no earlier than the year 2023 and no later than 2026 with an SN peak between 95 and 130. On the other hand, McIntosh et al. (2020), based on the identification of "termination" events that mark the end of the previous 11-yr sunspot cycle, the enhancement/acceleration of the present cycle, and the end of 22-yr magnetic activity cycle, found a relationship between the temporal spacing of terminators and the magnitude of sunspot cycles, providing the prediction for an SC 25 amplitude comparable with the top few since sunspot measurements began.

In this paper, we apply the method developed by Diego et al. (2010) to the geomagnetic activity indices measured during SC 24 to predict the activity level, as expressed by the SA proxy, to be expected in the current SC 25. In addition, we investigate the relationship between the recurrence index and the sunspot number to give the cycle amplitude to be compared with the other predictions. Finally, we provide information on the increasing rate of the forthcoming solar cycle and derive its shape and peak time. To this end, Section 2 presents the data used in this work and the basics of the method. Section 3 describes the application to the SN series and the obtained forecast for SC 25, whereas Section 4 discusses results and draws conclusions.

\section{Dataset and method}

The analysis performed in this paper is based on the method for identifying recurrent geomagnetic activity adopted in Diego et al. (2010). In order to apply the algorithm, we used daily data of geomagnetic index $a a$ (Mayaud, 1972) retrieved from http:// isgi.unistra.fr. The variability of solar activity has been evaluated on both sunspots areas (SA, daily data at https://www2. mps.mpg.de/projects/sun-climate) and the revised sunspot number (SILSO, 2019; Clette et al., 2014; url: http://www. sidc.be/silso/datafiles). The method had been developed as an improvement of those formerly suggested by Sargent (1985) and by Levitin et al. (1995). In particular, the Sargent (1985) algorithm was based on the $a a$ index autocorrelation between 27-d data series of adjacent Bartels rotations (BRs) and identified as recurrent periods those for which the correlation coefficient is greater than 0.2. Levitin et al. (1995) improved the Sargent (1985) algorithm by extending the computation up to three consecutive BRs. Nevertheless, these works did not provide quantitative information about the lifetime of the involved corotating solar structures that could last several BRs (Storini et al., 2006). In fact, the former methods were surely suitable to identify periods with the 27-day recurrent solar forcing, but these could not distinguish the effect due to a single long-lasting solar structure from a series of shorter-lasting ones. On the other hand, the persistence of solar corotating structures provides important information on the Sun's magnetic field evolution in the descending phase of a cycle, which can be assumed to be the preparation phase of the following one. For this reason, Diego et al. (2010) extended the recurrence index (called $I_{n}$ hereafter) up to seven BRs, $I_{n}$ being the sum of the correlation coefficient normalized over $n$ (varying from 1 to 6 ) terms as follow:

$$
I_{n}[a a]=\frac{1}{n} \sum_{i=1}^{n} r(N ; N-i)
$$

where $i$ is the number of BRs preceding the $N$ th one, $r$ is the correlation coefficient between the 27-day values of the aa index of the $N$-numbered BR and those of the $(N-i)$ th preceding one. The $I_{n}[a a]$ computation has been performed over more than a century of $a a$ index data in order to include a large number of solar cycles (i.e., 13 cycles from the 12th to the 24th). $I_{n}[a a]$ index gives information on the persistence of the geomagnetic activity 27-day recurrence. Since its amplitude depends on long-lived corotating structures on the Sun, such as coronal holes extending to low heliographic latitudes, $I_{n}[a a]$ shows low values, for high $n$, any time but not in case such solar structures are present (mainly during the descending phases of solar cycles). On the contrary, during solar activity maxima, these are not frequently observed at low latitudes, being mainly located at the solar poles, resulting in very low values of all the $I_{n}[a a]$. Thus, the highest values of the $I_{n}$ index, for high $n$, are characteristic of the descending phase of each cycle even though with different amplitudes. For instance, during some descending phases (e.g., cycle n. 22), the $I_{n}$ can decrease for increasing $n$ due to the reduced presence of long-lasting corotating regions on the Sun. In particular, the $I_{6}$ trend along the various cycles are the most suited to identify the presence of long-lived solar structures, as it has high values when those persisting up to 7 BRs are present, despite it might have values lower than the other $I_{n}[a a]$ (because if a structure persists for $7 \mathrm{BRs}$, it surely does for a lower number of BRs). On the contrary, the other $I_{n}[a a]$ are not sensitive to recurrences persisting more than $n+1$ BRs. The $I_{6}$ persistence has been characterized by computing its integral value over the descending phase of each cycle according to the start, maximum, and end time taken from Pesnell (2018).

The $I_{6}$ integrated values for each cycle have been correlated with the integrated values of the Sunspots Areas over the entire following cycle, obtaining a linear regression with a correlation coefficient of -0.83 . The average Root Mean Square Error (RMSE) of the regression reported in Figure 1 is about $2.2 \times 10^{4}$ (in unit of $10^{-6}$ solar surface hemisphere). RMSE is frequently used to measure the differences between values predicted by a model or an estimator and observed values (Hyndman \& Koehler, 2006). It has been computed according to the equation: 


$$
\operatorname{RMSE}=\sqrt{\frac{1}{n} \sum_{i=1}^{n}\left(\operatorname{Res}_{i}\right)^{2}}
$$

where $i$ is the solar cycle number, Res is the residual between observed and expected values. Figure 1 shows the linear regression between the two parameters. Note that the integrated SA values up to solar cycle 23 almost coincide (within $1 \%$ ) with those obtained by Diego et al. (2010), which used slightly different integration times taken from https://www. ngdc.noaa.gov/STP/SOLAR-DATA/SUNSPOT-NUMBERS/. In addition, the actual integrated sunspots areas over cycle 24 match, within the RMSE, the value predicted by Diego et al. (2010) at the end of cycle 23 (indicated by the green circle in Fig. 1) being the difference between the two values for SC 24 very small (about $1.5 \times 10^{3}$, i.e., lower than $2 \%$ above the observed value). As for the analysis performed in the previous work, the integrated value of $I_{6}$ allows the evaluation of the upcoming SC 25 amplitude. The red circle in Figure 1 represents the prediction of the sunspots areas over the entire next solar cycle. Considering the uncertainties, the SC 25 strength variation should encompass the observed ones of SCs 23, 21, 18 , and 17.

\section{Prediction of the sunspot number maximum}

In order to provide more fruitful information, the method described in the previous section has also been applied to the SN dataset. First, we performed a validation over SC 24, by computing the linear regression between $I_{6}$ and the $\mathrm{SN}$ maximum of the following cycle, over cycles from SC 13 to 23 green line in Figs. 1 and 2). Thus, we estimated the value for SC 24 to be compared with the observed one. The obtained correlation coefficient is -0.77 . The scatter plot and regression (green line) are illustrated in Figure 2. It can be seen that similarly to the case when SA (see previous section and Diego et al., 2010) is used, the forecasted (green circle) and observed values for the SN maximum of SC 24 are almost coincident, being their difference about 5 (about 121 predicted and 116 observed monthly value), thus within the RMSE of the regression and one standard deviation (SD) of the observed value (SD of the SC 24 is 8.2 as reported in http://www.sidc.be/silso/datafiles). Moreover, we repeated such correlation, including the actual SC 24 data point, thus obtaining the updated correlation coefficient of -0.77 . Hence we inferred that SC 25 should have an SN maximum of $205 \pm 29$ (red circle in Fig. 2), where the uncertainty has been computed as RMSE in equation (2). In order to check that $I_{6}$ is the most suitable $I_{n}$ to emphasize the geomagnetic persistence, as made for Sunspots Areas in Diego et al. (2010), we performed the correlation also with $I_{5}$ integrated values finding a worsening of correlation $(r=-0.74$; SN maximum $($ SC 25) $=194$; RMSE $=32$ ). The obtained prediction with $I_{6}$ encompasses the recorded SN maxima of SCs 22, 21, 18,17 , and, at the lower edge, also SCs 23 and 15. Note that SC 15 seems to be unusual as it sits at the edge of the error bar for SC 25 in Figure 2 (with a quite high SN peak), but it is much below in Figure 1 (with low sunspot areas), whereas all the other cycles seem far more consistent across the two figures, i.e., high SN peaks generally correspond to high sunspot

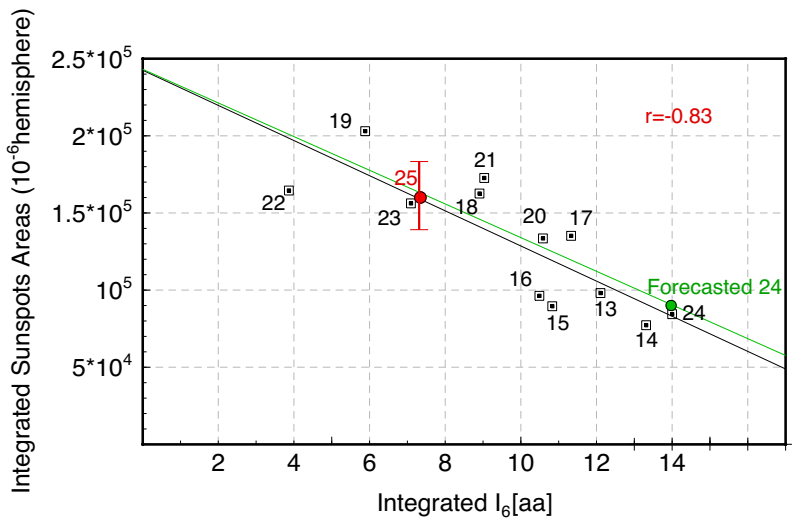

Fig. 1. Linear regression (black line) of $I_{6}[a a]$ integrated over the descending phase of each solar cycle versus the integrated sunspot area over the following cycle. The expected values of integrated sunspot area for SC 25 are represented by the red circle (confidence level is obtained as root mean square error). Green circle represents the SC 24 forecasted at the end of SC 23 by using the regression from Diego et al. (2010) (represented by the green line) over SCs from n. 14 to 23 .

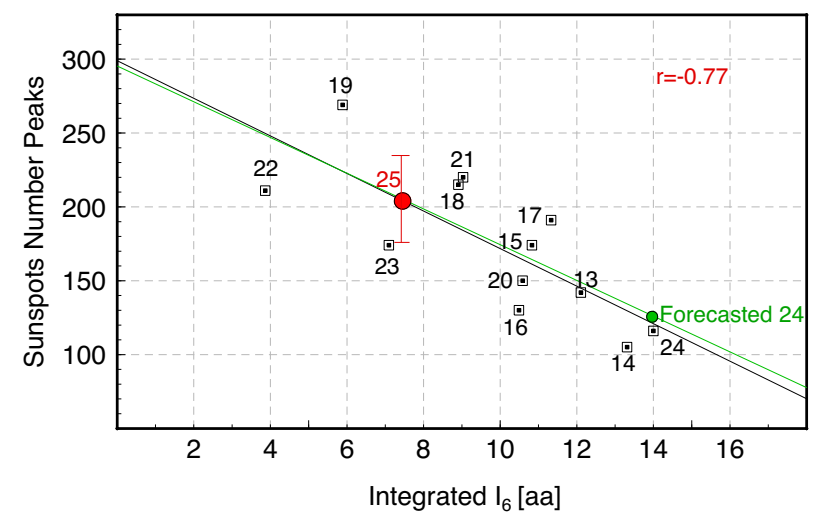

Fig. 2. As in Figure 1, the linear regression of $I_{6}[a a]$ integrated over the descending phase of each solar cycle versus the sunspot peaks of the following cycle. The expected peak of SC 25 is represented by the red circle (confidence level is obtained as root mean square error). Green circle represents SC 24 forecasted by using the regression computed over SCs from 14 to 23 (represented by the green line).

areas. A possible explanation could be an exception to the general trend for low amplitude cycles to be preceded by a solar cycle transition with a high number of spotless days and vice versa (https://wwwbis.sidc.be/silso/spotless). In this respect, SC 15 has a quite high amplitude cycle, although preceded by a solar cycle transition with a high number of spotless days, which could explain the observed low value of the integrated sunspot areas, despite the high sunspot number peak.

In order to evaluate also the SC 25 rising time from minimum to sunspot maximum, we exploited the well-known Waldmeier effect (Waldmeier, 1935). As a matter of fact, the maximum sunspot amplitude is known to be inversely correlated to the cycle rise time (time interval between the onset and the maximum of each cycle) or correlated to its highest growth rate (the increasing rate of $\mathrm{SN}$ before the peak) 


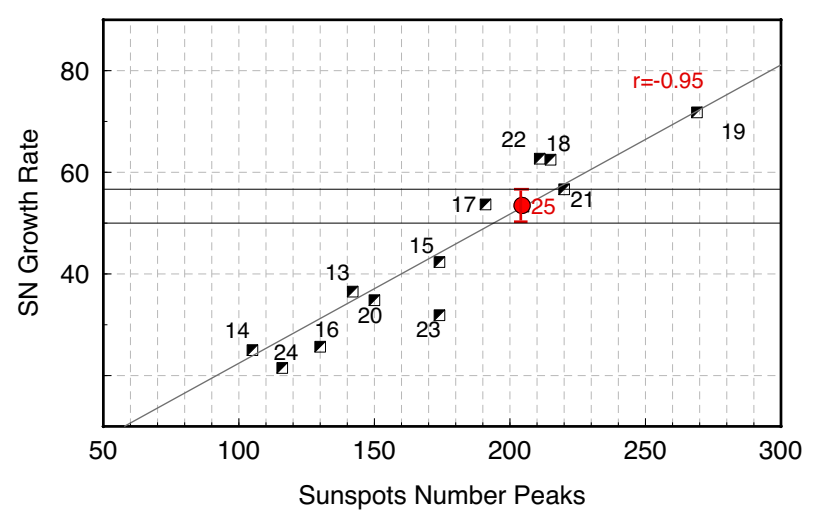

Fig. 3. Linear regression between $\mathrm{SN}$ growth rate and $\mathrm{SN}$ peaks. The growth rate forecasted for the SC 25 is $53 \pm 5$, thus comparable with those of SCs 17 and 21.

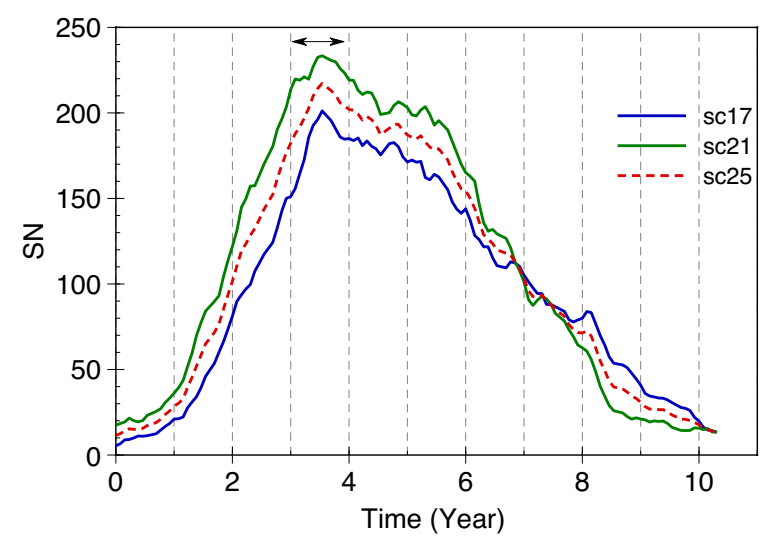

Fig. 4. Comparison between SC 17 and SC 21 by using a 12 Bartelsrunning mean values. The trend of SC 25 is derived as the average values of SCs 17 and 21. The maximum is expected between 3 and 4 years after the cycle onset (black arrow).

(Waldmeier, 1935). These effects are called WE1 and WE2, respectively. Past works found that the WE1 effect occurs only for even-numbered cycles (Dikpati et al., 2008) or under specific algorithm settings (Karak \& Choudhuri, 2010). On the contrary, the WE2 seems to be commonly accepted to be a robust result found in different kinds of theoretical models, such as the flux transport dynamo models, which currently appear to be amongst the most promising for explaining the solar cycle (see Karak \& Choudhuri, 2011, and reference therein). Thus, we used the WE2 approach to retrieve the forthcoming SN growth rate to provide an indication of the shape of the next SC 25 . The growth rate has been computed as $\Delta \mathrm{SN} / \Delta t$, being $\Delta t$ the time interval between minimum and the following maximum, and $\triangle \mathrm{SN}$ the corresponding $\mathrm{SN}$ variation (13-month smoothed data from http://www.sidc.be/silso/cyclesminmax). Figure 3 shows a good correlation $(r=-0.95)$ between the $\mathrm{SN}$ maximum and the highest increasing rate during the ascending phase of each cycle from SC 13 to 24 . These quantities are highly correlated even considering the uncertainties in the minima time detection that could be addressed to some cycles. Thus we can infer the growth rate for SC 25 by assuming the SN maximum of 205 as predicted above. The estimated value is about $53 \pm 5 \mathrm{yr}^{-1}$ which can identify similar features among the various cycles.

It can be noted in Figure 3 that the increasing rate of SC 25 is very close to that of cycles 17 and 21 . Once again, the error bar has been computed as RMSE in equation (2). Figure 4 shows a direct comparison between SC 17 and 21 with a very similar profile, especially in the rising phase, as expected from the values of their growth rates and amplitudes. Thus, we derived the SC 25 time profile (dashed red line in Fig. 4) as an average of the SCs 17 and 21, rescaled to have the SN maximum equal to $205 \pm 29$. The expected maximum can be foreseen about 3.5 years after the cycle onset (i.e., about mid2023). A closer inspection of Figure 4 suggests that a second SN maximum might appear, after a $\sim 6$ month period corresponding to the so-called Gnevyshev Gap (e.g., Storini et al., 2003; Vecchio et al., 2010; Laurenza et al., 2012) from 4 to 4.5 years (i.e., around the end of 2023/first half of 2024).

\section{Conclusion}

We studied the recurrences in geomagnetic activity using the $I_{6}$ index, expressing the autocorrelation of the $a a$-index up to seven consequent Bartels rotations over the period 18782019 (i.e., solar cycles n. 12-24). The observed $I_{6}$ enhanced values were exploited to develop a solar activity activity prediction algorithm, which is correlated with the SA and SN datasets. The results of the analysis provide a twofold contribution to the prediction of the solar cycle strength. First, the effective amplitude of SC 24 turned out to be almost coincident with that estimated from SC 23 as checked for both sunspots number and integrated areas. In fact, the relative difference between the predicted and observed values are lower than the Standard Deviation of the SC 24 peak. Second, the highly reliable results obtained for SC 24 for both sunspot number maximum and areas integrals allow for a complete prediction of the SC 25 . Following the Waldmeier (WE2) approach, we also derived a rough estimation of the forthcoming cycle shape and $\mathrm{SN}$ maximum time, based on the good correlation found between the SN growth rate and the SN maximum. Since WE2 provides an estimation of the highest value of the increasing rate, the predicted time to reach the SN maximum could be underestimated. Therefore, the correlation shown in Figure 3 has to be considered just as an useful tool to identify similar shapes of previous cycles to address the next SC 25 . Figure 5 displays the $I_{6}$ time series along with SN since cycle $n$. 12. The shape of SC 25 has been obtained, as for Figure 4, by properly merging those of SCs 17 and 21 selected as the most similar according to the predicted increasing rate and amplitude. Therefore, combining the peak amplitude with the increasing rate, we can estimate that the SC 25 will have its first peak around mid-2023 with an amplitude of about $205 \pm 29$.

As far as space climate-related long-term variations are concerned, we observe that SC 24 deviated downwards from the Modern Maximum cycles (after 1950, Hathaway, 2010). Two similar intercycle decreases in activity occurred in SCs 5-6 and 14, which were closer analogs of the recent SC 24. These were identified as the two previous Gleissberg minima centuries ago. The circumstance that a new Gleissberg minimum was already overdue suggests that it coincided with 


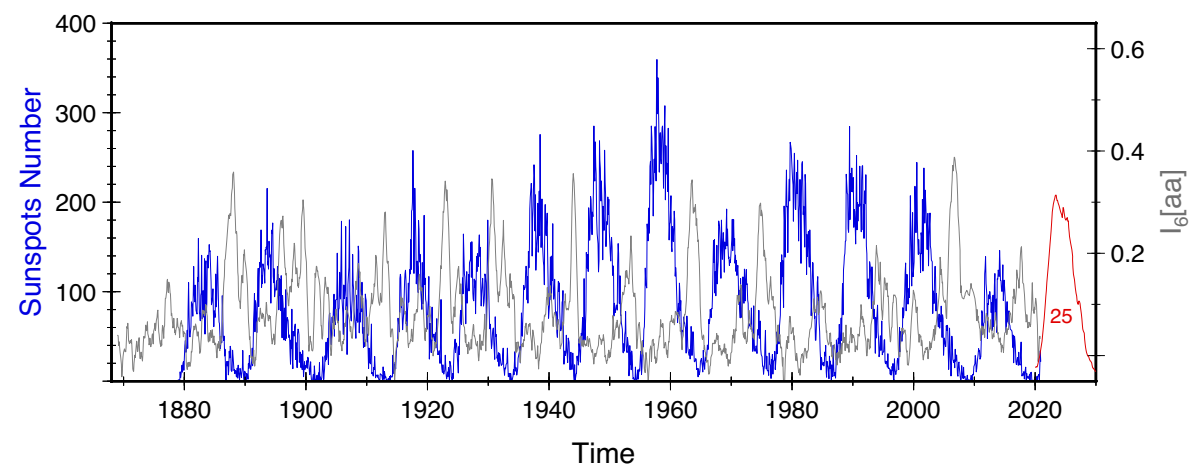

Fig. 5. Monthly SN since solar cycle 12 (blue line) along with $I_{6}[a a]$ (grey line). The cycle 25 profile (red line) is obtained with the predicted values of maximum peak and growth rate from regressions in Figures 2 and 3, respectively.

SC 24. This is corroborated by the following considerations: (i) the Gleissberg cycle on scales 60-130 yr has been found to have a relevant role in the grand minima generation (Vecchio et al., 2017, 2019); (ii) the activity decrease during SC 24 occurs on a timescale comparable to the Gleissberg cycle period after SC 14, when the sunspot areas have been observed to have the lowest peak value in the last century (Svalgaard et al., 2005; Diego et al., 2010); (iii) the Schwabe cycle behavior between cycles 23 and 24 was found to be similar to that observed in the Maunder and Dalton minima, thus suggesting a minimum-like behavior. Indeed, the Schwabe cycle period of SC 23 was longer than 11 years, as observed during anomalous periods such as the Maunder and Dalton minima (Fligge et al., 1999; Usoskin \& Mursula, 2003; Petrovay, 2010). As a matter of fact, the mean SC length value from minimum to a minimum is 11.03 years (median 11.25 years) with a standard deviation of $1.18 \mathrm{yr}$, as computed by using the recalibrated sunspot number (Pesnell, 2018) over SCs from 1 to 23. On the other hand, the length of SC 23 was 12.3 years, i.e., just above one standard deviation (corresponding to a probability of $27 \%$ ), similarly to the higher than average length of SCs 4 and 5 (13.5 and $12.6 \mathrm{yr}$, respectively) and 13 (11.9 yr) preceding grand minima periods. In addition, a violation of the Gnevyshev-Ohl rule (solar cycles are paired in even-odd pairs so that the odd cycle is more intense than the preceding even cycle) occurred for the recent SCs 22-23, preceding SC 24 when the activity noticeably decreased. Such a violation also heralded some previous minima (Komitov \& Bonev, 2001; Usoskin et al., 2021), possibly due to a phase catastrophe in solar cyclic evolution accompanying a quick change of solar activity from a high to a low level (Usoskin et al., 2021). Thus we speculate that SC 24 coincided with a Gleissberg minimum, after which solar activity is expected to increase, in line with our prediction for SC 25.

Acknowledgements. The results presented in this paper rely on geomagnetic indices calculated and made available by ISGI Collaborating Institutes from data collected at magnetic observatories. We thank the involved national institutes, the INTERMAGNET network, and ISGI (http://isgi.unistra.fr). The authors also thank the Italian Space Agency for the financial support under the contracts ASI "LIMADOU Scienza+" and "Hemera". The editor thanks two anonymous reviewers for their assistance in evaluating this paper.

\section{References}

Bhowmik P, Nandy D. 2018. Prediction of the strength and timing of sunspot cycle 25 reveal decadal-scale space environmental conditions. Nat Commun 9: Article ID 5209. https://doi.org/10.1038/ s41467-018-07690-0.

Bushby P, Tobias S. 2007. On Predicting the solar cycle using meanfield models. Astrophys J 661: 1289-1296. https://doi.org/ $10.1086 / 516628$.

Cameron RH, Dikpati M, Brandenburg A. 2017. The Global Solar Dynamo. Space Sci Rev 210: 367-395. https://doi.org/10.1007/ s11214-015-0230-3.

Charbonneau P. 2010. Dynamo models of the solar cycle. Living Rev Sol Phys 7: Article ID 3. https://doi.org/10.12942/lrsp2010-3.

Clette F, Svalgaard L, Vaquero JM, Cliver EW. 2014. Revisiting the sunspot number. A 400-year perspective on the solar cycle. Space Sci Rev 186(1-4): 35-103. https://doi.org/10.1007/s11214-0140074-2.

Diego P, Storini M, Laurenza M. 2010. Persistence in recurrent geomagnetic activity and its connection with Space Climate. J Geophys Res 115: A06103. https://doi.org/10.1029/2009JA014716.

Dikpati M, Gilman PA, De Toma G. 2008. The Waldmeier effect: an artifact of the definition of Wolf sunspots number? Astrophys $J$ 673: 99-101. https://doi.org/10.1086/527360.

Feynman J. 1982. Geomagnetic and solar wind cycles, 1900-1975. J Geophys Res 87(A8): 6153-6162. https://doi.org/10.1029/ JA087iA08p06153.

Fligge M, Solanki SK, Beer J. 1999. Determination of solar cycle length variations using the continuous wavelet transform. $A \& A$ 346: $313-321$.

Hathaway DH. 2010. The solar cycle. Living Rev Sol Phys 7(1): 65. https://doi.org/10.1007/lrsp-2015-4.

Hathaway DH, Wilson RM. 2006. Geomagnetic activity indicates large amplitude for sunspot cycle 24. Geophys Res Lett 33: L18101. https://doi.org/10.1029/2006GL027053.

Hyndman RJ, Koehler AB. 2006. Another look at measures of forecast accuracy. Int J Forecast 22(4): 679. https://doi.org/ 10.1016/j.ijforecast.2006.03.001.

Karak BB, Choudhuri AR. 2010. The Waldmeier effect in sunspot cycles. Department of Physics, Indian Institute of Science, Bangalore, India. arXiv:1008.2931v1 [astro-ph.SR].

Karak BB, Choudhuri AR. 2011. The Waldmeier effect and the flux transport solar dynamo, Department of Physics. Mon Not R Astron Soc 410: 1503-1512. https://doi.org/10.1111/j.1365-2966.2010. 17531.x. 
Komitov B, Bonev B. 2001. Amplitude variations of the 11 year cycle and the current solar maximum 23. Astrophys $J$ 554: L119L122. https://doi.org/10.1086/320908.

Laurenza M, Vecchio A, Storini M, Carbone V. 2012. Quasi-biennial modulation of galactic cosmic rays. Astrophys J 749: 167 (11 pp). https://doi.org/10.1088/0004-637X/749/2/167.

Levitin AE, Obridko VN, Val'chuk TE, Golyshev SA, Dremukhina LA. 1995. Recurrence index of planetary geomagnetic activity and its connection with the solar rotation. Geomag Aeron 35: 260-263.

Mayaud PN. 1972. The aa indices: A 100-year series characterizing the magnetic activity. J Geophys Res 77(34): 6870-6874. https://doi.org/10.1029/JA077i034p06870.

McIntosh SW, Chapman S, Leamon RJ, Egeland R, Watkins NW, et al. 2020. Overlapping magnetic activity cycles and the sunspot number: Forecasting sunspot cycle 25 amplitude. Sol Phys 295: 163. https://doi.org/10.1007/s11207-020-01723-y.

Ohl AI. 1966. Wolf's number prediction for the maximum of the cycle20. Soln Dann 12: 84

Pesnell WD. 2008. Predictions of solar cycle 24. Sol Phys 252: 209-220. https://doi.org/10.1007/s11207-008-9252-2.

Pesnell WD. 2016. Predictions of solar cycle 24: How are we doing? Space Weather 14: 10-21. https://doi.org/10.1002/2015SW001304.

Pesnell WD. 2018. Effects of version 2 of the International Sunspot Number on naïve predictions of solar cycle 25. Space Weather 16: 1997-2003. https://doi.org/10.1029/2018SW002080.

Pesnell WD, Schatten K. 2018. An early prediction of the amplitude of solar cycle 25. Sol Phys 293: 112. https://doi.org/10.1007/ s11207-018-1330-5.

Petrovay K. 2010. Solar cycle prediction. Living Rev Sol Phys 7: 59. Article ID 6. https://doi.org/10.12942/lrsp-2010-6.

Petrovay K. 2020. Solar cycle prediction. Living Rev Sol Phys 17: Article ID 2. https://doi.org/10.1007/s41116-020-0022-z.

Sargent HH. 1985. Recurrent geomagnetic activity: Evidence for long-lived stability in solar wind structure. J Geophys Res 90: 1425-1428. https://doi.org/10.1029/JA090iA02p01425.

Schatten K. 2005. Fair space weather for solar cycle 24. Geophys Res Lett 32: L21106. https://doi.org/10.1029/2005GL024363.

Schatten KH, Scherrer PH, Svalgaard L, Wilcox JM. 1978. Using Dynamo Theory to predict the sunspot number during solar cycle 21. Geophys Res Lett 5: 411-414. https://doi.org/10.1029/ GL005i005p00411.
SILSO. 2019. World data center - sunspot number and long-term solar observations. Royal Observatory of Belgium, on-line sunspot number catalogue: http://www.sidc.be/SILSO/, years 1878-2020.

Storini M, Bazilevskaya GA, Fluckiger EO, Krainev MB, Makhmutov VS, Sladkova AI. 2003. The Gnevyshev gap: A review for space weather. Adv Space Res 31: 895-900. https://doi.org/ 10.1016/S0273-1177(02)00789-5.

Storini M, Hofer MY, Sykora J. 2006. Towards the understanding of coronal hole occurrence during the Schwabe cycle. Adv Space Res 38: 912-920. https://doi.org/10.1016/j.asr.2006.03.024.

Svalgaard L, Cliver E, Kamide Y. 2005. Sunspot Cycle 24: Smallest cycle in 100 years? Geophys Res Lett 32: 5. https://doi.org/ 10.1029/2004GL021664.

Tobias S, Hughes D, Weiss N. 2006. Unpredictable Sun leaves researchers in the dark. Nature 442: 26. https://doi.org/10.1038/ 442026c.

Upton LA, Hathaway DH. 2018. An updated solar cycle 25 prediction with AFT: The modern minimum. Geophys Res Lett 45: 8091-8095. https://doi.org/10.1029/2018GL078387.

Usoskin I, Mursula K. 2003. Long-term solar cycle evolution: review of recent developments. Sol Phys 218: 319-343. https://doi.org/ 10.1023/B:SOLA.0000013049.27106.07.

Usoskin I, Kovaltsov G, Kiviaho W. 2021. Robustness of solar-cycle empirical rules across different series including an updated ADF sunspot group series. Sol Phys 296: Article ID 13. https://doi.org/ 10.1007/s11207-020-01750-9.

Vecchio A, Laurenza M, Carbone V, Storini M. 2010. The quasibiennial modulation of solar neutrino flux, solar and galactic cosmic rays by the solar cyclic activity. Astrophys J Lett 709: L1-L5. Article ID 1. https://doi.org/10.1088/2041-8205/709/1/L1.

Vecchio A, Lepreti F, Laurenza M, Alberti T, Carbone V. 2017. Connection between solar activity cycles and grand minima generation. $A \& A$ 599: 12. Article ID A58. https://doi.org/10.1051/ 0004-6361/201629758.

Vecchio A, Lepreti F, Laurenza M, Carbone V, Alberti T. 2019. Solar activity cycles and grand minima occurrence. N. Cim. C 42: Article ID 15. https://doi.org/10.1393/ncc/i2019-19015-0.

Waldmeier M. 1935. Neue Eigenschaften der Sonnenfleckenkurve. Astron Mitt Eidgenoss Sternwarte Zurich 14: 105-136.

Cite this article as: Diego P \& Laurenza M 2021. Geomagnetic activity recurrences for predicting the amplitude and shape of solar cycle n. 25. J. Space Weather Space Clim. 11, 52. https://doi.org/10.1051/swsc/2021036. 